\title{
Cinétique de la production d'ascospores de Sclerotinia trifoliorum Eriks en chambre de culture et en conditions climatiques naturelles. Incidences pratiques et épidémiologiques
}

\author{
G Raynal \\ avec la collaboration technique de F Ferrari et M Mouret
}

INA-PG, INRA, Laboratoire de pathologie végétale, 78850 Thiverval-Grignon, France

(Reçu le 16 mars 1990; accepté le 10 juin 1990)

\begin{abstract}
Résumé - La production en ascospores d'apothécies de Sclerotinia trifoliorum est évaluée quotidiennement au laboratoire sous diverses conditions. Elle dépend de l'âge et du diamètre des apothécies. Elle est maximale pour des apothécies de 7 à $12 \mathrm{j}$. La sporulation moyenne, durant leur vie entière, d'apothécies de 4 à $5 \mathrm{~mm}$ de diamètre est de 1,4 $\times 10^{6}$ ascospores. La température optimale est de $15{ }^{\circ} \mathrm{C}$, les apothécies demeurant alors sporulantes pendant 18 à $20 \mathrm{j}$. A $5{ }^{\circ} \mathrm{C}$ et $10^{\circ} \mathrm{C}$, la sporulation est plus faible, mais les apothécies demeurent fonctionnelles plus d'un mois. À $25^{\circ} \mathrm{C}$, elles meurent en quelques jours. La sporulation est meilleure à la lumière qu'à l'obscurité. Des apothécies séparées de leurs sclérotes et mises en conditions d'HR satisfaisantes conservent un fonctionnement normal. Soumises au climat naturel, les apothécies projettent leurs ascospores de fin octobre à début décembre, comme l'indiquent des piégeages réalisés avec un capteur par aspiration. La sporulation est la plus forte par temps doux et très humide. Elle est faible en conditions anticycloniques. La projection des ascospores est presque nulle la nuit. Elle débute vers $10 \mathrm{~h}$, est maximale vers $15-16 \mathrm{~h}$ et cesse vers $19 \mathrm{~h}$. Outre leur intérêt pour une meilleure production d'ascospores utilisables en contaminations artificielles, ces observations précisent les facteurs agissant sur le développement de la sclérotiniose des légumineuses fourragères.
\end{abstract}

Sclerotinia trifoliorum / apothécies / ascospores / piégeage / climat

Summary - Kinetics of the ascospore production of Sclerotinia trifoliorum Eriks in growth chamber and under natural climatic conditions. Practical and epidemiological incidence. Sclerotia of Sclerotinia trifoliorum produced in the laboratory were placed under favourable conditions for capogenesis at varying temperatures in the light or in the dark. Ascospores discharged by apothecia were counted on a daily basis throughout the life cycle of the apothecia, ie 18-20 d at $15^{\circ} \mathrm{C}$. The quantity of spores collected depended on the diameter and age of the apothecia. The greatest discharge was generally when apothecia were 7-12 d old (fig 1). The mean number of ascospores collected from apothecia over the entire life cycle was $1.4 \times 10^{6}$ from apothecia 4-5 mm in diameter and $4.7 \times 10^{6}$ for apothecia $7 \mathrm{~mm}$ in diameter. Temperature directly affected sporulation. Spore discharge was abundant at $25^{\circ} \mathrm{C}$, but lasted only a few hours and the apothecia died within a few days. At $5-10^{\circ} \mathrm{C}$ discharge was weak, but lasted over 1 month. At 20 and in particular at $15^{\circ} \mathrm{C}$, discharge was optimal, with sporulation continuing for a mean of 3 weeks (fig 2). Light was more conducive to sporulation than dark. Apothecia that had become detached from their stipes maintained normal sporulation and responded to temperature in the same way as apothecia attached to sclerotia. However, they survived for a somewhat shorter period (fig 3). Sclerotia produced in the laboratory then subjected to natural climatic conditions in the soil from the beginning of September began to produce apothecia in mid-October. Ascospores that were discharged were collected with a vacuum spore trap (fig 4). In 1987 and 1988, ascospore discharge was studied from the end of October to the beginning of December, ie the period during which the apothecia remained alive. During that time constantly wet soil resulted in normal functioning of the apothecia. Spore discharge was maximal when the weather was mild $\left(10-15^{\circ} \mathrm{C}\right.$ ) and very humid (ie, fog, drizzle or rain). Discharge was slight under anticyclonic conditions when the nights were cold and afternoons sunny. Slight frost did not inhibit further sporulation once conditions became favourable again (fig 5). Ascospore discharge was almost non-existent at night, especially when temperatures were low. Discharge usually began about $10.00 \mathrm{~h}$, was maximum between 15.00 and $16.00 \mathrm{~h}$ and ceased at about $19.00 \mathrm{~h}$ (table I and fig 6). This periodicity was related to temperature. The present study has resulted in an enhanced yield of ascospores from apothecia produced in the laboratory for artificial inoculation of red clover in screening for resistance. It has also better defined some important epidemiological factors affecting crown rot of forage legumes. 


\section{INTRODUCTION}

Sclerotinia trifoliorum Eriks est le principal responsable du manque de pérennité des cultures de trèfle violet dans les régions tempérées et nordiques, par suite des pourritures hivernales et printanières qu'il provoque. II entraîne des dégâts du même type chez la luzerne, mais généralement moins prononcés. Dans les conditions naturelles, les différents auteurs ont montré que l'inoculum primaire est constitué d'ascospores disséminées à partir des apothécies qui se constituent en fin d'automne à partir des sclérotes formés sur les plantes mortes au printemps précédent (Valleau et al, 1933; Raynal, 1985).

Après avoir étudié les principaux facteurs agissant sur la production des apothécies (Raynal, 1987), nous maîtrisons bien maintenant la production en grande quantité des apothécies en conditions contrôlées, afin de disposer à tout moment d'un inoculum pour la sélection de trèfles violets résistants à la sclérotiniose. Aucune donnée, par contre, n'a été publiée à ce jour sur la sporulation de $S$ trifoliorum, ni au champ, ni en chambre de culture. Cette lacune peut avoir plusieurs raisons : difficulté, voire impossibilité d'une étude suivie pratiquée dans les conditions du champ, récolte ou obtention souvent aléatoires des apothécies naturelles ou produites en chambre de culture afin d'étudier en détail leur sporulation. De telles informations sont dispersées pour les autres Sclerotinia, en particulier pour $S$ sclerotiorum, pour lequel aucun article n'est consacré en totalité à ce sujet. C'est pourquoi, disposant d'une méthode nous assurant une production abondante d'apothécies utilisables à toutes fins, nous avons entrepris en 1987 et 1988 l'étude de l'évolution de leur sporulation tout au long de leur vie. Les manipulations, faites en conditions contrôlées puis sous les conditions climatiques naturelles, ont eu pour but de préciser une phase importante de l'épidémiologie de la sclérotiniose des légumineuses fourragères, et ont permis d'accroître le rendement des apothécies produites par la suite, en routine, au laboratoire.

\section{SPORULATION EN CHAMBRE DE CULTURE, EN «MINISERRES»}

\section{Matériel et méthodes}

Les sclérotes de $S$ trifoliorum sont obtenus à partir de notre isolat de référence $\mathrm{ST} 78.02$, par culture à $20^{\circ} \mathrm{C}$ pendant 45 j d'implants mycéliens en boîtes de Roux à l'obscurité, sur fragments de pommes de terre autoclavés. Après récolte, les sclérotes sont placés pendant 1 mois en étuve à $30^{\circ} \mathrm{C}$, afin de lever leur dormance. Puis on les dépose dans de la vermiculite maintenue constamment humide, en "Miniserres" (marque BHR) à $15^{\circ} \mathrm{C}$. Rappelons que les "Miniserres" sont constituées d'une terrine de $45 \times 30 \times 8 \mathrm{~cm}$, recouverte d'un capot en polystyrène transparent et posée sur une soucoupe rectangulaire destinée à recevoir de l'eau. Ces conditions sont celles que nous utilisons pour induire une production massive d'apothécies (Raynal, 1987).

\section{Évolution et sporulation durant toute leur vie d'apothécies individualisées à $15^{\circ} \mathrm{C}$ et à la lumière continue}

Dès que les sclérotes ont formé leurs stipes, c'est-àdire 1 mois environ après leur dépôt sur vermiculite, on en prélève 20 , sans les désinfecter, qui portent 1 seul stipe d'une longueur voisine de $1 \mathrm{~cm}$, prêt à différencier une apothécie. Ces sclérotes peuvent porter ou non d'autres ébauches de stipes. Si, par la suite, ces stipes tendent à évoluer en apothécies, on les supprime pour ne conserver que l'apothécie d'origine, afin de ne pas être gêné par des sporulations annexes. On place chaque sclérote dans un cristallisoir de $\varnothing 90 \mathrm{~mm}$ et on l'englobe dans une épaisseur de 1 $\mathrm{cm}$ d'eau gélosée en surfusion (15 g/l d'agar-agar), en prenant soin de maintenir le stipe dressé, son extrémité dépassant de la surface de la gélose.

Après refroidissement, l'ensemble est retourné audessus d'un couvercle de boîte de Petri en polystyrène. Le retournement du cristallisoir sur le couvercle suffit à garder tout au long de la manipulation une humidité saturante dans l'enceinte, qui se manifeste par une condensation sur les parois. On porte les critallisoirs en chambre climatisée à répartition homogène de la température, réglée à $15 \pm 1^{\circ} \mathrm{C}$, à $30 \mathrm{~cm}$ audessus d'une source lumineuse fortement ventilée éclairant en continu (lumière fluorescente blanche, 1200 lux). On satisfait ainsi aux conditions de température et d'éclairement nécessaires à une évolution normale des stipes, puis des apothécies, les sclérotes étant placés dans un milieu riche en eau et les stipes et apothécies restant constamment dans une atmosphère saturée d'humidité, à la lumière (Raynal, 1987).

Au fur et à mesure de leur maturation, les asques projettent leurs ascospores qui se déposent sur les couvercles. Pour quantifier la sporulation, on récupère quotidiennement les couvercles et on les remplace aussitôt par des neufs. Le comptage des ascospores se fait en utilisant un hématimètre, après leur mise en suspension dans 5 à $10 \mathrm{ml}$ d'eau permutée, selon leur quantité, en frottant les couvercles avec un pinceau. De plus, chaque jour, on mesure le diamètre des apothécies à l'aide d'une loupe binoculaire équipée d'un micromètre, afin d'évaluer le nombre d'ascospores émises par $\mathrm{mm}^{2} \mathrm{~d}$ apothécie et par jour.

On procède aux mesures et aux dénombrements sur chacune des apothécies, jusqu'à leur mort. En rai- 
son du temps requis pour ces opérations, il n'a pas été possible de les pratiquer sur plus de 20 apothécies.

\section{Effet de la température sur la sporulation d'apothécies formées}

Des sclérotes porteurs chacun d'un stipe sont tout d'abord récupérés pour la suite des opérations, et conservés pour évolution sur vermiculite humide, sous lumière blanche continue, à $15^{\circ} \mathrm{C}$. Lorsque les apothécies sont âgées de $8 \mathrm{j}$, on prélève délicatement les sclérotes porteurs d'une apothécie d'un diamètre voisin de 6 à $7 \mathrm{~mm}$ environ. On les fixe alors dans de la gélose par groupes de 5 , dans un cristallisoir fermé par un couvercle de boîte de Petri, comme décrit plus haut. On les porte ensuite en chambre climatisée éclairée équivalente à celle utilisée précédemment à $5,10,15,20$ et $25^{\circ} \mathrm{C}$. Les dénombrements d'ascospores et mesures des diamètres des apothécies sont faits comme précédemment, jusqu'à ce que les apothécies ne sporulent plus. Vingt apothécies, réparties en 4 cristallisoirs, sont utilisées par température.

\section{Effet de la lumière ou de l'obscurité continue sur la sporulation, à $15^{\circ} \mathrm{C}$}

Vingt apothécies d'environ $7 \mathrm{~mm}$ de diamètre, solidaires de leurs sclérotes et âgées de 8 j, sont fixées dans de la gélose par groupes de 5 en 4 critallisoirs, et placées en chambre climatisée éclairée déjà décrite, à $15^{\circ} \mathrm{C}$. Un lot similaire est mis à l'obscurité dans une boîte cartonnée hermétiquement close, placée dans la même enceinte climatisée.

Après $72 \mathrm{~h}$ de lumière ou d'obscurité continue, on dénombre les ascospores projetées et on mesure les diamètres des apothécies.

\section{Sporulation à $15^{\circ} \mathrm{C}$ et à la lumière d'apothécies détachées de leurs sclérotes}

Les réserves nutritives des sclérotes servent sans nul doute à la constitution des apothécies, cela étant démontré simplement par la relation directe entre la grosseur des sclérotes et le nombre et le diamètre des apothécies qu'ils portent (Raynal, 1983). Mais, une fois les apothécies constituées et ayant atteint leur taille quasi définitive, c'est-à-dire $8 \mathrm{j}$ après leur naissance,on peut se demander si, en plus de son rôle trophique, le sclérote ne transfère pas des substances pouvant agir sur les phénomènes de sporulation. Pour avoir des éléments de réponse, nous avons sectionné les stipes à la base des apothécies, afin de séparer ces dernières de toute relation avec le sclérote qui leur a donné naissance.

Les apothécies ainsi traitées sont placées dans des boîtes de Petri en polystyrène, ces boîtes contenant une épaisseur de $5 \mathrm{~mm}$ de gélose (15 g d'agar/l d'eau permutée) que l'on coule au moment de l'emploi. Les apothécies, d'un diamètre voisin de $7 \mathrm{~mm}$, sont pla- quées à raison de 5 par boîte contre la gélose encore peu solidifiée, leur face sporulante tournée vers le couvercle. Après solidification complète de la gélose, les boîtes sont placées de la même façon que précédemment en chambre climatisée et éclairée, à 5,10 , 15,20 et $25^{\circ} \mathrm{C}$. On procède quotidiennement au dénombrement des ascospores libérées et à la mesure des diamètres des apothécies jusqu'à la fin de la sporulation.

\section{Résultats}

\section{Évolution et sporulation durant toute leur vie d'apothécies individualisées à $15^{\circ} \mathrm{C}$ et à la lumière}

Lorsque l'on part de sclérotes de taille équivalente $(5$ à $7 \mathrm{~mm})$ porteurs chacun d'un seul stipe, on constate une grande variabilité dans les dimensions des apothécies qui se forment. Ainsi, pour les 20 sclérotes testés, le diamètre des apothécies au moment de leur expansion maximale va de 1,3 à $7,4 \mathrm{~mm}$, avec une moyenne de $4,3 \mathrm{~mm}$ (écart type 1,5 mm). La répartition des apothécies selon leur diamètre est la suivante : $1,3-2,0 \mathrm{~mm}=25 \% ; 2,0-4,0 \mathrm{~mm}$ $=35 \% ; 4,0-6,0 \mathrm{~mm}=30 \% ; 6,0-7,4=10 \%$. Les plus petites apothécies (diamètre $<2 \mathrm{~mm}$ ) ont une durée de vie ne dépassant pas $10 \mathrm{j}$. Elles sporulent peu ou pas du tout. Pour les autres, on constate que leur diamètre maximal est atteint en 8 à $12 \mathrm{j}$. Après avoir atteint un maximum, le diamètre régresse légèrement jusqu'à la mort de l'apothécie, qui se manifeste par un brunissement puis un affaissement. Elle survient généralement entre les $18^{\circ}$ et $2^{\circ} \mathrm{j}$. Les petites apothécies se dégradent le plus vite, alors que la durée de vie des plus grosses dépasse $23 \mathrm{j}$.

La sporulation débute dès que le diamètre de l'apothécie est supérieur à 1,5 mm en moyenne, c'est-à-dire le plus souvent dès le lendemain de la mise en place des sclérotes porteurs de stipes dans les cristallisoirs. Les apothécies ont alors une forme de pilon, dont seule la partie invaginée sporule. La récolte quotidienne d'ascospores atteint son maximum entre les $7^{e}$ et $12^{e} j$ pour la plupart des apothécies, les plus grosses sporulant abondamment plus tard, entre les $13 e$ et $17 \mathrm{e}$ j (pour 2 apothécies de $7 \mathrm{~mm}$ de diamètre, $115 \times 10^{3}$ ascospores/apothécie/jour du $7^{\mathrm{e}}$ au $12^{\mathrm{e}} \mathrm{j}$ et $440 \times 10^{3}$ du $13^{\mathrm{e}}$ au $17^{\mathrm{e}} \mathrm{j}$ ). La quantité de spores récoltées quotidiennement varie considérablement selon la taille et l'âge des apothécies : de 0 pour les quelques apothé- 
cies dont le diamètre se maintient inférieur à 1,5 $\mathrm{mm}$, à $736 \times 10^{3}$ au maximum pour celles de diamètre voisin de $7 \mathrm{~mm}$.

Dans ces conditions, la quantité de spores récoltées par apothécie est elle aussi éminemment variable, et la présentation des moyennes journalières des récoltes de l'ensemble des 20 apothécies n'aurait pas grande signification. C'est pourquoi nous ne rapporterons ici que les résultats obtenus avec des apothécies de diamètre supérieur à $3 \mathrm{~mm}$ le $8^{\mathrm{e}} \mathrm{j}$, soit 12 apothécies. La figure 1 rapporte les résultats de 2 types d'apothécies : celles, majoritaires, dont le diamètre est compris entre 4 et $5 \mathrm{~mm}$ (diamètre moyen 4,7 ; écart type 0,9 ) et la sporulation de 2 apothécies de 6 à $7,4 \mathrm{~mm}$. Sur toute la durée de leur vie (13 à $23 \mathrm{j}$ ), on récolte par apothécie et selon leur taille $240 \times 10^{3}$ à $4,7 \times 10^{6}$ ascospores (moyenne : $1,42 \times 10^{6}$, écart type $1,28 \times 10^{6}$ pour l'ensemble des 12 apothécies).

La quantité de spores par $\mathrm{mm}^{2}$ d'apothécie par jour est également extrêmement variable d'une apothécie à l'autre et au cours du temps. Si l'on considère globalement les 12 apothécies citées précédemment, le minimum est de $2,1 \times$ $10^{3}$ le $5^{e} \mathrm{j}$ et le maximum $7,7 \times 10^{3}$ le $10^{0} \mathrm{j}$. De plus, pendant la période de sporulation active, il ne semble exister aucune relation entre l'âge ou la taille des apothécies et la quantité de spores émises par unité de surface, qui oscille autour d'une moyenne journalière de $4,9 \times 103 / \mathrm{mm}^{2}$. Pour un petit nombre d'apothécies, indifférenciables visuellement des autres, cette valeur

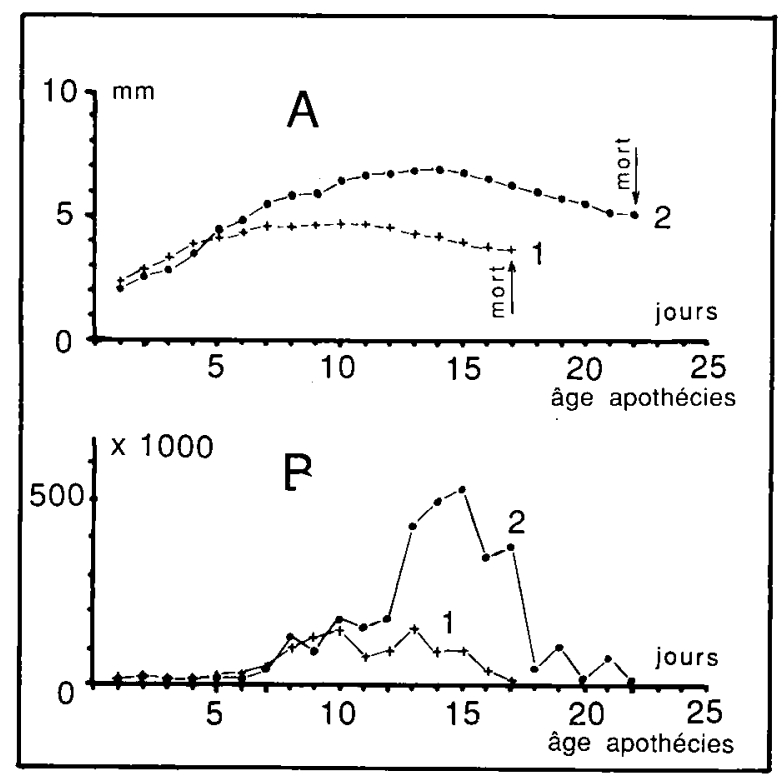

Fig 1. Évolution au cours du temps des caractéristiques et sporulation moyennes des apothécies de diamètres 4,0-5,0 $\mathrm{mm}$ (courbes 1) et de diamètre 6,0-7,4 $\mathrm{mm}$ (courbes 2), à $15^{\circ} \mathrm{C}$ et sous lumière fluorescente blanche continue. A : croissance diamétrale, $\mathrm{B}:$ nombre d'ascospores récoltées. peut être, certains jours, très élevée, proche de $20 \times 10^{3}$, sans que l'on puisse fournir d'explication à ce phénomène, et revenir le lendemain à la normale. En fin de sporulation, la sporulation par unité de surface prend le plus souvent des valeurs très basses.

On retiendra de ces résultats l'extrême variabilité individuelle des apothécies pour leur aptitude à sporuler. La conclusion de cette première étude est que si l'on veut comparer valablement l'effet de divers facteurs sur la sporulation, il ne faut pas partir de stipes qu'on laisse évoluer en apothécies, mais d'apothécies déjà formées, susceptibles de sporuler convenablement en conditions favorables. C'est pourquoi dans ce qui suit nous n'avons utilisé que des apothécies de 8 $\mathrm{j}$ ayant atteint un diamètre de 6 à $7 \mathrm{~mm}$.

\section{Effet de la température sur l'évolution d'apothécies formées, en lumière continue}

Les variations de sporulation sont encore loin d'être négligeables d'un lot d'apothécies à l'autre, mais sans commune mesure avec ce que nous avons décrit au paragraphe précédent, les effets individuels étant plus ou moins tamponnés par le groupement par 5 des apothécies et, surtout, par la similitude de leurs diamètres $(6,0$ à $7,6 \mathrm{~mm})$. Toutefois, les écarts types, notamment pour les quantités quotidiennes de spores émises, atteignent des valeurs comprises entre 10 et $50 \%$ de la moyenne de chaque traitement.

La figure 2 montre l'évolution de la sporulation au cours du temps en fonction de la température. La quantité de spores émise par $\mathrm{mm}^{2}$ d'apothécie varie dans le même sens en raison de la quasi-constance des surfaces des apothécies, leurs diamètres ne diminuant que très faiblement en cours d'essai, quelle que soit la température. Ces quantités ne seront donc pas rapportées ici. II est clair que la température conditionne directement la durée de vie des apothécies, déjà âgées de $8 \mathrm{j}$ en début d'essai, et leur intensité de sporulation. À $25^{\circ} \mathrm{C}$, la sporulation est immédiatement intense, mais s'arrête très vite, la mort des apothécies survenant en 4 à $5 \mathrm{j}$. À 5 et $10^{\circ} \mathrm{C}$, la quantité de spores émises est relativement faible, mais dure longtemps, les apothécies étant toujours vivantes au $17^{\mathrm{e}} \mathrm{j}$ d'expérimentation (âgées de $25 \mathrm{j}$ ). La sporulation est relativement abondante à $20^{\circ} \mathrm{C}$ et le rendement encore meilleur à $15^{\circ} \mathrm{C}$, les apothécies subsistant longtemps à ces températures. Ces résultats sont en accord avec ceux trouvés pour la différenciation des stipes en apothécies (Raynal, 1987). 


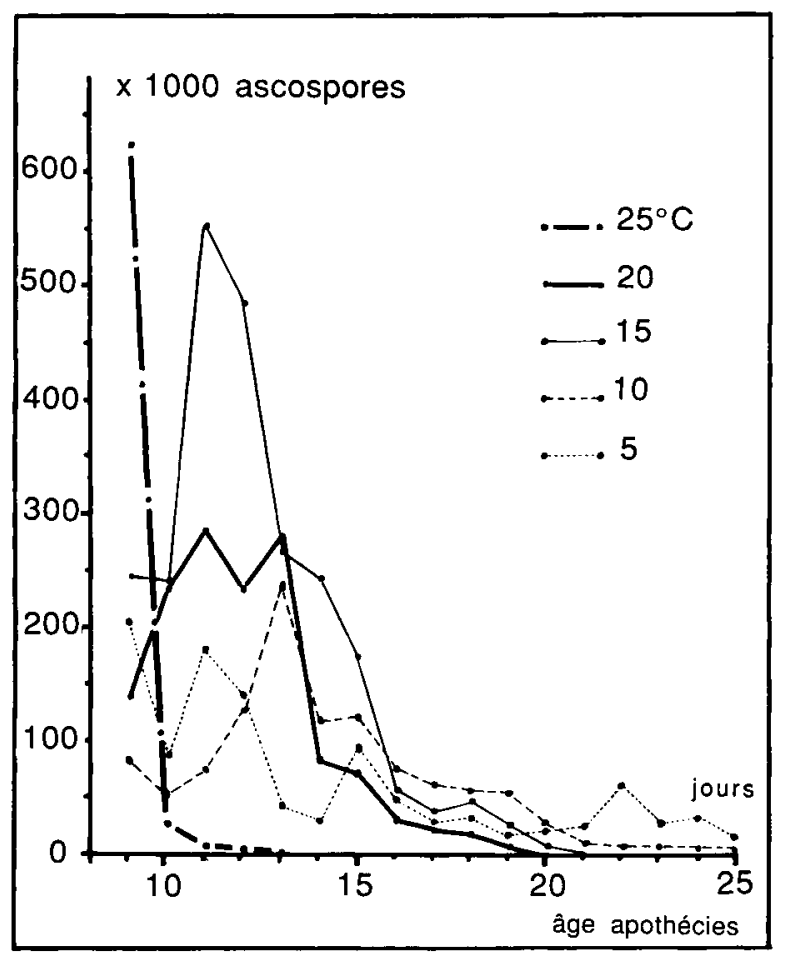

Fig 2. Sporulation moyenne selon la température, en lumière continue, d'apothécies âgées de $8 \mathrm{j}$ au début de l'essai et mesurant 6 à $7 \mathrm{~mm}$ de diamétre, élevées primitivement à $15^{\circ} \mathrm{C}$.

\section{Effet de la lumière et de l'obscurité continues sur la sporulation, à $15^{\circ} \mathrm{C}$}

En faisant les mêmes remarques que précédemment concernant la variabilité des résultats d'un lot d'apothécies à l'autre, on observe pour un même traitement que la quantité d'ascospores récoltée pendant 3 j consécutifs est pratiquement 3 fois plus élevée à la lumière qu'à l'obscurité : 491000 contre 158000 par apothécie. Ceci est à rapprocher des résultats concernant l'influence de la lumière sur la diftérenciation des stipes en apothécies, où nous avions trouvé que l'obscurité continue empêche les stipes de se transformer (Raynal, 1987). Les apothécies ayant, avant l'essai, reçu un éclairement continu de 8 j nécessaire à leur évolution, celles portées à l'obscurité ont pu bénéficier, pendant les 3 j de l'essai, de l'effet de la période préalable d'éclairement. Des expérimentations complémentaires devraient être faites pour définir la durée minimale d'éclairement nécessaire à l'induction de la décharge des ascospores, ainsi que la rémanence d'action de l'éclairement.

\section{Sporulation à $15^{\circ} \mathrm{C}$ et à la lumière d'apothécies détachées de leurs sclérotes}

Les mêmes remarques que précédemment s'imposent pour la variabilité des résultats. La figure 3 indique la quantité d'ascospores récoltée quoti-

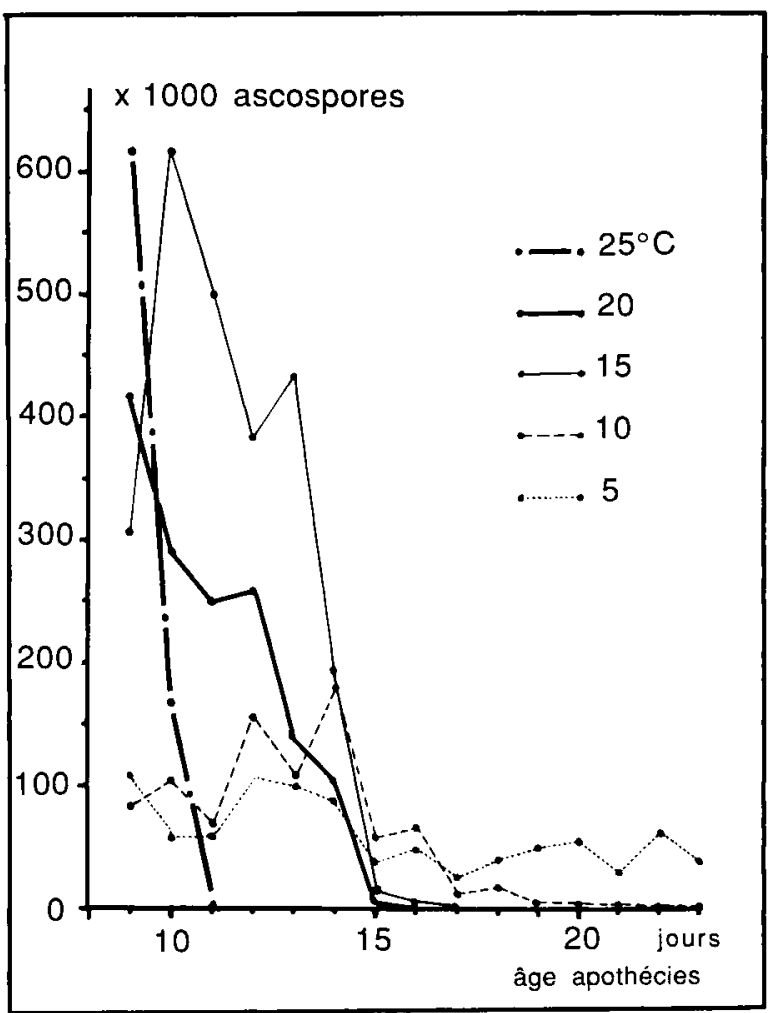

Fig 3. Sporulation moyenne selon la température, en lumière continue, d'apothécies âgées de $8 \mathrm{j}$ au début de l'essai, d'un diamètre de 6 à $7 \mathrm{~mm}$, séparées de leur sclérote.

diennement par apothécie selon la température. Les courbes sont très voisines de celles de la figure 2. Cependant, la durée de vie des apothécies séparées de leur sclérote est légèrement moins longue que lorsqu'elles y restent attachées. Mais ni la quantité de spores émises, ni la répartition dans le temps de la sporulation ne sont profondément modifiées. Une fois différenciées, et à condition que l'eau ne constitue pas un facteur limitant, les apothécies possèdent donc suffisamment de réserves pour poursuivre normalement leur existence sans l'aide du sclérote dont elles sont issues. Remarquons toutefois qu'à la fin de leur vie, les apothécies plaquées contre la gélose sont plus ou moins envahies de bactéries et se recouvrent d'une poudre blanche due aux conidies de la forme asexuée Myrioconium sp. Ces phénomènes n'étaient pas visibles sur les apothécies attachées à leur sclérote et dressées au-dessus de la surface de la gélose.

\section{SPORULATION EN CONDITIONS CLIMATIQUES NATURELLES}

\section{Matériel et méthodes}

Nous avons étudié la sporulation d'apothécies produites dans des conditions semi-naturelles, en captu- 
rant quotidiennement les ascospores projetées à l'aide d'un aspirateur de spores, d'octobre à décembre 1987 et de novembre à décembre 1988. Pour des raisons pratiques, il ne nous a pas été possible de capturer les ascospores dans un champ de trèfle naturellement contaminé : éloignement du laboratoire rendant impossibles les relevés quotidiens, difficulté à faire fonctionner correctement sur une longue période un capteur de spores alimenté par des batteries, conditions climatiques locales et de l'année pouvant entraîner des sorties irrégulières d'apothécies.

C'est pourquoi nous avons placé des sclérotes de $S$ trifoliorum en terre près du laboratoire, à proximité d'une source électrique de $220 \mathrm{~V}$, permettant de faire fonctionner aisément et de façon régulière le capteur. Les sclérotes sont produits au laboratoire, comme pour les études précédentes. Après avoir passé 1 mois à $30^{\circ} \mathrm{C}$, ils sont mis, début septembre, dans des terrines contenant de la terre de jardin, les terrines étant elles-mêmes enfoncées dans le sol du jardin botanique de Grignon et livrées aux conditions climatiques naturelles. Les premières apothécies sont apparues le 16 octobre 1987 et le 23 octobre 1988. Les dates de ces sorties correspondent parfaitement aux observations que nous avions réalisées lors d'essais précédents, en conditions semi-naturelles (Raynal, 1983) et au champ (Raynal, 1985). Les sclérotes porteurs d'apothécies sont alors déterrés délicatement et rassemblés de façon à obtenir un lot de 50 jeunes apothécies. On dispose les sclérotes dans une petite terrine dont le fond comporte de nombreuses perforations, remplie de terre de jardin, selon un rectangle de 2 à $10 \mathrm{~cm}$, en les enfouissant légèrement dans la terre et en prenant soin de ne pas casser les apothécies. La terrine est enfoncée dans le sol et le capteur de spores est placé juste au-dessus de la ligne d'apothécies. Le sol de la terrine n'est pas arrosé, l'eau du sol environnant suffisant à la maintenir humide de la fin octobre au début de décembre.

Le capteur, de fabrication personnelle, est basé sur le principe de l'appareil de Hirst (1952), repris par d'autres auteurs (Taris, 1968; Rapilly, 1970). II nous a servi par ailleurs, avec un montage approprié, à étudier l'émission des ascospores d'Epichloe typhina (Raynal et Clinkspoor, 1988). II se compose principalement d'un cylindre enregistreur de thermographe dont le tambour effectue un tour en $24 \mathrm{~h}$ grâce à son mécanisme d'horlogerie. Le système d'aspiration est constitué d'une turbine de climatisation de voiture (Renault 4), fonctionnant normalement sur une batterie de $12 \mathrm{~V}$. Disposant d'une source proche de $220 \mathrm{~V}$, nous avons interposé un chargeur de batterie fournissant un courant de $12 \mathrm{~V}$ et réglé sur $2 \mathrm{~A}$. Le tambour d'enregistrement est placé sous un capot cylindrique en polyéthylène facile à ôter, comportant une fente de 90 $\times 2 \mathrm{~mm}$, et relié au système d'aspiration par un tuyau souple de $4 \mathrm{~cm}$ de diamètre. Au réglage de $2 \mathrm{~A}$, la vitesse d'aspiration de l'air au niveau de la fente est de $4 \mathrm{~m} . \mathrm{sec}^{-1}$, ce qui correspond à un débit de $2,59 \mathrm{~m}^{3} \cdot \mathrm{h}^{-}$ 1 , mais n'est plus que de $25 \mathrm{~cm} \cdot \mathrm{sec}^{-1}$ à $5 \mathrm{~cm}$ de la fente (vitesses mesurées au thermoanémomètre à fil chaud EBRO, type EB 4003, diffusion par Biobloc, Paris).
Une feuille en plastique transparent, sur laquelle sont tracées des lignes verticales définissant des intervalles de $3 \mathrm{~h}$, est fixée au tambour rotatif. On enduit la feuille d'une très fine pellicule d'un mélange de lanoline + vaseline assurant l'adhésion des microparticules aspirées. La fente d'aspiration est placée à $5 \mathrm{~cm}$ audessus des apothécies (fig 4). La température et l'hygrométrie sont enregistrées à proximité des apothécies. Les conditions météorologiques locales sont notées. Les relevés pluviométriques (pluie ou rosée) sont ceux de la station INRA de bioclimatologie, distante de quelques centaines de mètres.

On change la feuille de plastique toutes les $24 \mathrm{~h}$ et on procède au comptage des ascospores aspirées qui s'y sont collées. Pour cela, on découpe la feuille en bandes de $3 \mathrm{~h}$. On lave ces bandes, en les frottant avec un pinceau dans une quantité connue d'eau (5 à $10 \mathrm{ml}$ ) additionnée d'un mouillant (Tween 20). Les ascospores, rapidement mises en suspension dans l'eau, sont comptées au microscope sur un hématimètre.

\section{RÉSULTATS}

\section{Intensité de la sporulation en fonction des conditions climatiques}

La figure 5 montre la quantité d'ascospores récoltées quotidiennement en fonction du temps et des conditions climatiques. Pour ne pas alourdir les graphiques, les variations journalières de l'hygrométrie de l'air, mesurée à $20 \mathrm{~cm}$ de la surface du sol, ne sont pas indiquées, bien que nous les ayons enregistrées quotidiennement. En effet, elles dépendent des conditions atmosphériques et de l'humidité du sol. Sauf en cas de gel, ce dernier est resté constamment humide ou saturé d'eau pendant les périodes considérées. Par temps anticyclonique, le ciel peut être brumeux

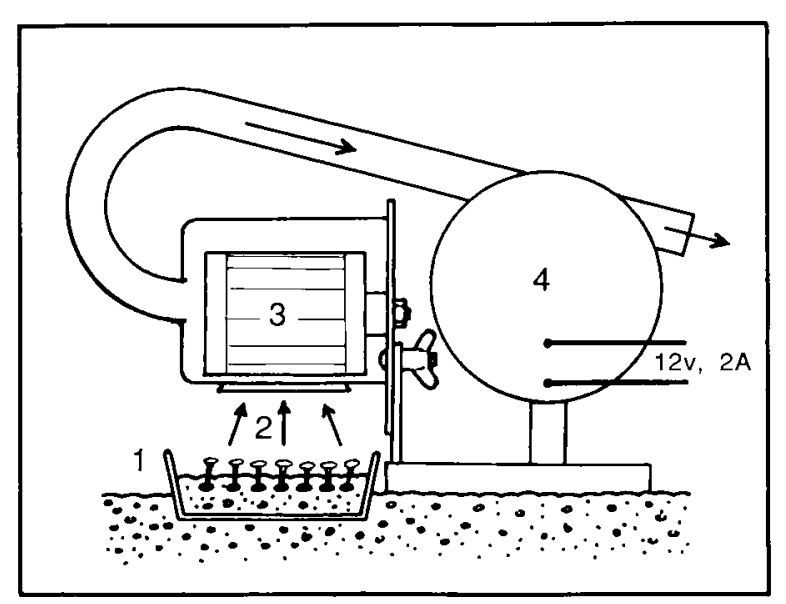

Fig 4. Schéma du capteur d'ascopores par aspiration, en place au-dessus d'apothécies de $S$ trifoliorum. 1 : terrine contenant les sclérotes porteurs d'apothécies; 2 : fente d'aspiration; 3 : tambour d'enregistrement portant une feuille de plastique enduite de lanoline + vaseline; 4 : turbine d'aspiration alimentée en 12 volts et 2 ampères. 
toute la journée ou clair, au moins l'après-midi, avec un bon ensoleillement. Dans ce cas, l'humidité relative de l'air peut descendre aux environs de $50 \%$ entre 14 et $15 \mathrm{~h}$. Elle remonte vite à la saturation dès 16 à $18 \mathrm{~h}$, et s'y maintient jusqu'au lendemain vers 10 ou $12 \mathrm{~h}$, tant que les conditions anticycloniques persistent. Par brouillard, bruine et pluie, l'humidité de l'air à proximité du sol est égale à $100 \%$, aussi bien le jour que la nuit. Cela explique que les apothécies n'aient jamais souffert d'aucune sécheresse, ni du sol, ni de l'air, et qu'elles aient pu accomplir normalement leur cycle de sporulation.

D'après les graphiques dont l'allure est très similaire pour les 2 années, on peut dégager les conclusions générales suivantes :
- Dans les conditions climatiques naturelles du Bassin parisien, l'émission des ascospores a lieu de la fin octobre jusqu'au début du mois de décembre au moins, la quantité de spores récoltées diminuant avec l'âge des apothécies. Les apothécies demeurent vivantes plus d'un mois, ce qui correspond bien aux observations faites précédemment en chambre de culture à des températures basses et à nos notations au champ (Raynal, 1985). Notons que les apothécies sont mortes en 1987 du 10 au 14 décembre, à la suite d'une période de gel assez prononcé. En 1988, leur mort a eu lieu du 3 au 10 janvier, mais leur sporulation, vérifiée 1 fois par semaine à partir du 5 décembre, était constamment faible $\left(5 \times 10^{4}\right.$ à $10^{5}$ ascospores/jour

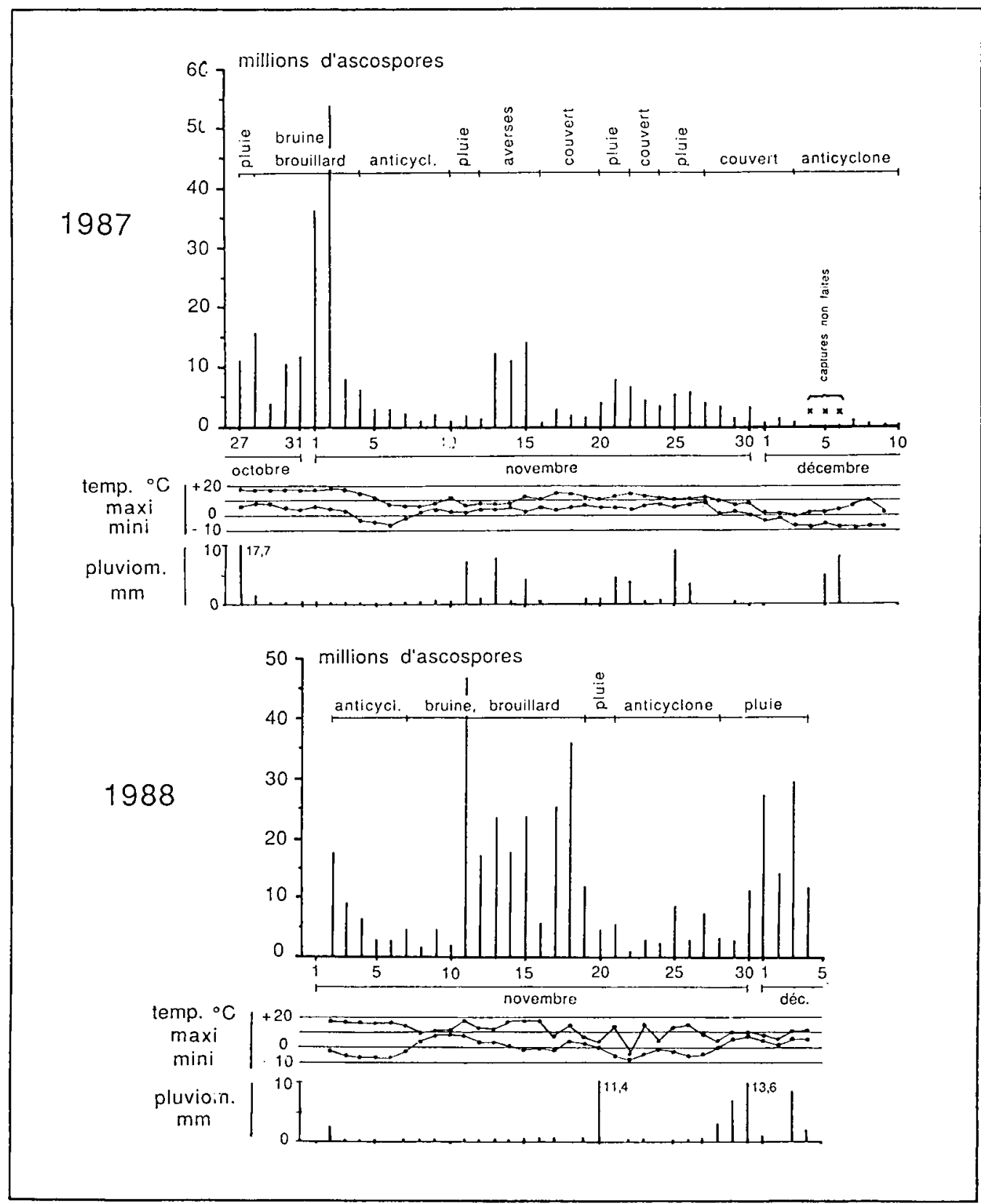

Fig 5. Quantité d'ascospores projetées par 50 apothécies soumises aux conditions climatiques naturelles de 1987 et 1988 , et récoltées chaque jour avec un capteur de spores par aspiration. 
pour l'ensemble du lot). En cours d'expérimentation, aussi bien en 1987 qu'en 1988, quelques apothécies sont mortes, mais d'autres, nées des sclérotes, les ont remplacées, si bien que le total de 50 a pu être maintenu.

- Par brouillard persistant, bruine ou pluie, les températures sont généralement clémentes, avec des écarts thermiques réduits entre le jour et la nuit. II s'ensuit que les conditions les plus propices à la sporulation se rencontrent dans ces périodes, surtout lorsque la température diurne avoisine $15^{\circ} \mathrm{C}$. Le brouillard et la bruine, malgré le très faible volume de précipitations qu'ils occassionnent, semblent plus favorables que la pluie à la projection des ascospores. En effet, lors de pluies, les apothécies, du fait de leur forme concave, retiennent de l'eau sur leur hyménium, ce qui doit gêner la projection directe des ascospores. Pour le vérifier, nous avons réalisé une expérience complémentaire en plaçant des sclérotes porteurs d'apothécies en cristallisoirs, sur vermiculite humide à $15^{\circ} \mathrm{C}$ et à la lumière. On apporte ou non à l'aide d'un pinceau de l'eau permutée additionnée de mouillant (Tween 20 à 1\%) à la surface de l'hyménium des apothécies et on place aussitôt au-dessus d'elles des lames de microscopie. Les cristallisoirs sont fermés par un couvercle en polystyrène transparent. Seules les apothécies qui n'ont pas reçu d'eau sporulent normalement. Les autres n'éjectent aucune ascospore tant que leur surface reste recouverte d'un film d'eau, soit, dans les conditions de l'essai, durant environ 1 h. Dès que la pellicule d'eau s'est évaporée, les apothécies reprennent une sporulation normale. Ce phénomène est identique à celui signalé sur $S$ sclerotiorum par Kruger (1975) et Lamarque et Rappilly (1981), chez lequel la présence d'eau sur l'hyménium empêche la libération des ascospores. Pour le premier auteur, de fortes pluies pourraient même lessiver les ascospores émises dans le film d'eau et diminuer ainsi l'incidence de la sclérotiniose sur le colza, ce qui serait à vérifier chez les légumineuses fourragères.

- En conditions anticycloniques, la sporulation est faible, sans doute à cause de la sécheresse de l'air pendant la période journalière de sporulation (cf paragraphe suivant) et des températures nocturnes, souvent fortement négatives, qui pourraient ralentir la formation et la maturation des asques.

- De faibles gelées nocturnes ou matinales ne gênent pas la sporulation si les températures et surtout l'humidité atmosphérique sont favorables durant la journée. Si les apothécies ne sont pas trop âgées, elles supportent aisément une période de quelques nuits de gel, et sont capables de sporuler dès que les conditions redeviennent favorables.

Notons que les dimensions moyennes des apothécies, mesurées à la mi-novembre, sont plus faibles en 1987 (4,7 mm de diamètre, écart type 1,4) qu'en $1988(6,7 \mathrm{~mm}$, écart type 2,5). Les surfaces totales des apothécies, calculées en fonction de leurs diamètres réels, sont de 948 $\mathrm{mm}^{2}$ en 1987 (1) et $1656 \mathrm{~mm}^{2}$ en 1988 (2), soit un rapport (2)/(1) de 1,76. En regard, les quantités totales d'ascospores récoltées en novembre 1987 sont de $214 \times 10^{6}$ (1) et de $332 \times 10^{6}$ pour la même période en 1988 (2), soit un rapport (2)/ (1) de 1,55. Cette quasi-équivalence reflète la constance du fonctionnement des apothécies lorsque les conditions climatiques sont analogues (ce fut le cas en novembre 1987 et 1988 où le nombre et la durée des périodes favorables à la sporulation ont, par chance, été assez semblables). Elle entre, de plus, dans les limites de la variabilité des captures de spores, variabilité que nous n'avons pas testée directement, faute de posséder d'autres capteurs, mais que l'on peut pressentir au vu des expérimentations en chambre de culture.

\section{Rythme journalier de projection des ascospores}

Le tableau I montre la remarquable régularité de la projection des ascospores sur une durée de $24 \mathrm{~h}$, quelle que soit la période de sporulation considérée. À titre d'exemple, nous donnons les résultats pour des périodes d'abondante sporulation, mais on observe la même répartition lorsque les apothécies projettent peu d'ascospores: presque nulle la nuit, la projection d'ascospores est maximale l'après-midi.

Malgré de grandes variations journalières pour les captures totales (cf fig 4), la répartition des captures en cours de journée est très homogène. Des comptages horaires de spores entre $9 \mathrm{~h}$ et $18 \mathrm{~h}$ (heure légale, soit UT +1 ), effectués sur 11 j répartis entre le 3 et le 30 novembre 1987, ont montré que les captures débutent au plus tôt à $10 \mathrm{~h}$, parfois à $12 \mathrm{~h}$ (heure légale). Le maximum des captures est atteint entre 15 et $16 \mathrm{~h}$, rarement entre 14-15 h ou 16-17 h.

II est vraisemblable que la température constitue un facteur essentiel qui règle la projection des ascospores car, outre son propre effet, elle agit sur l'humidité relative de l'air et sur la durée 
Tableau I. Quantités d'ascospores (en milliers) récoltées en moyenne sur 24 heures pendant les périodes de sporulation les plus actives de 50 apothécies (voir fig 4), selon des tranches tri-horaires (heures légales, soit TU +1 ).

Année Période de sporulation o $h-3 h \quad 3 h-6 h \quad 6 h-9 h \quad 9 h-12 h \quad 12 h-15 h \quad 15 h-18 h \quad 18 h-21 h \quad 21 h-24 h$

\begin{tabular}{|c|c|c|c|c|c|c|c|c|c|}
\hline \multirow[t]{2}{*}{1987} & 3 octobre - 3 novembre & 22 & 4 & 16 & 216 & 6780 & 6560 & 16 & 12 \\
\hline & 13 au 15 novembre & 26 & 47 & 39 & 1240 & 6230 & 5670 & 72 & 82 \\
\hline 1988 & 11 au 19 novembre & 37 & 66 & 48 & 1850 & 4590 & 5525 & 62 & 58 \\
\hline & 30 novembre -4 décembre & 23 & 29 & 22 & 760 & 5885 & 2765 & 68 & 52 \\
\hline
\end{tabular}

de mouillage des apothécies. Elle atteint son maximum vers $15 \mathrm{~h}$, moment de la plus forte projection des ascospores. Basse en matinée, en soirée et surtout la nuit (rappelons qu'à la minovembre, à la latitude de Grignon, le soleil se lève à $8 \mathrm{~h}$ et se couche à $17 \mathrm{~h}$ ), elle ne permet pas cette éjection, ou rarement et de façon peu intense. Si l'on se rapporte, d'après nos enregistrements, à l'évolution journalière de l'hygrométrie de l'air mesurée à une dizaine de $\mathrm{cm}$ audessus du sol, on voit qu'elle se maintient égale à $100 \%$ de $18 \mathrm{~h}$ à $10 \mathrm{~h}$. Par contre, de $10 \mathrm{~h}$ à 18 $h$, elle varie selon les conditions atmosphériques : elle peut rester très élevée (80 à 100\%) par temps couvert, de bruine ou de pluie, ou descendre très bas (50 à 60\%) vers $15 \mathrm{~h}$ en conditions anticycloniques. Or c'est pendant cette période que se déroule la sporulation, qui est la plus abondante lorsque l'hygrométrie est élevée. Une hygrométrie basse ne fait que ralentir la sporulation, sans toutefois la stopper complètement, ce qui est conforme aux observations de Lamarque et Rapilly (1981) chez $S$ sclerotiorum.

Signalons enfin que la projection des ascospores, telle que nous l'observons sur les feuilles de plastique lanolinées, n'est pas continue à l'intérieur des diverses tranches horaires. On distingue constamment, en effet, d'étroites bandes plus denses que d'autres en spores, comme en témoigne la figure 6 . Ces bandes ne résultent pas, nous l'avons vérifié, d'un fonctionnement discontinu du capteur de spores. Nous ne les avons d'ailleurs jamais observées lors de nos captures d'ascospores d'Epichloe (Raynal et Clinkspoor, 1988). Elles ont pour origine la projection synchrone de très nombreuses ascospores pendant des temps plus ou moins longs, évalués à $5 \mathrm{~min}$ pour les bandes les plus étroites et jusqu'à 10 min pour les plus larges, ces projec- tions étant suivies de phases de repos où les apothécies éjectent moins d'ascospores. Ce mécanisme, qu'il conviendrait d'étudier en détail, pourrait associer les processus de maturation des asques et diverses actions physiques (impacts et gouttes de pluie, vent, microturbulences, etc) pour déclencher la projection des ascospores. On sait, par exemple, que si l'on exerce une dépression brutale au-dessus d'un lot d'apothécies, elles projettent presque instantanément un nuage d'ascospores, qui correspond à la décharge simultanée des asques mûrs. Ce phénomène, également bien connu chez $S$ sclerotiorum («puffing»), aiderait, en créant des microturbulences, à la dispersion des ascospores (Newton, Sequeira, 1972; Steadman, 1983).

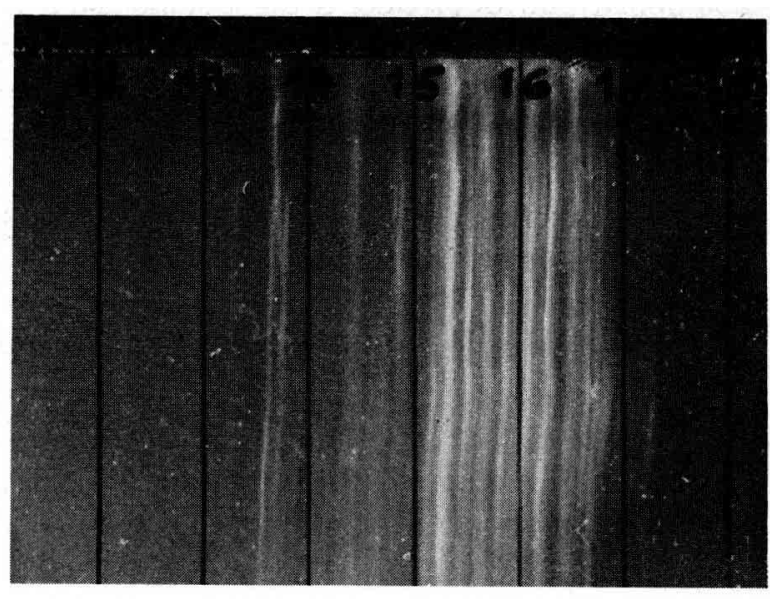

Fig 6. Feuille de plastique enduite de lanoline + vaseline montrant les bandes blanches des ascospores capturées de $12 \mathrm{~h}$ à $18 \mathrm{~h}$ (heure légale $=\mathrm{UT}+1$ ) . 


\section{DISCUSSION ET CONCLUSION}

Les modalités de sporulation de $S$ trifoliorum et de $S$ sclerotiorum présentent des analogies. Ainsi, une apothécie de $S$ sclerotiorum peut éjecter en conditions favorables environ $2 \times 10^{6}$ ascospores pendant la durée de sa vie, qui atteint en moyenne $9 \mathrm{j}$ (Schwartz et Steadman, 1978). Une forte teneur en eau du sol est nécessaire pour que les apothécies de $S$ sclerotiorum émettent des ascospores, ces apothécies n'étant pas fonctionnelles si un film d'eau libre les recouvre (Lamarque et Rapilly, 1981). L'optimum de température est cependant plus élevé chez $S$ sclerotiorum $\left(22{ }^{\circ} \mathrm{C}\right.$ au lieu de $15^{\circ} \mathrm{C}$ pour $S$ trifoliorum), mais on retrouve l'action néfaste des fortes températures ( 28 et $32{ }^{\circ} \mathrm{C}$ ), qui entraînent en quelques heures la mort des apothécies, alors que les températures basses leur conservent longtemps leur aptitude à la sporulation (Newton et Sequeira, 1972). On ne sait pas, par contre, s'il existe chez $S$ sclerotiorum un rythme journalier de sporulation, tel que nous l'avons montré chez $S$ trifoliorum.

Sur un plan pratique, nos observations en chambre de culture ont permis d'améliorer de façon notable le rendement en ascospores des apothécies que nous produisons en routine pour la contamination des trèfles violets lors des tests de résistance variétale. C'est ainsi qu'en conservant la même technique de récolte, mise au point par Tourvieille et al (1978) - on coupe les apothécies, on les plaque sur des couvercles de boîtes de Petri et, en se désséchant à la température ordinaire, elles libèrent leurs ascospores nous sommes passés d'un rendement moyen de 30000 ascospores par apothécie (Raynal, 1983) à 50000 (Raynal, 1987) et maintenant à 200000 à 300000 . Ceci a été obtenu simplement en surveillant visuellement la maturation des apothécies dans leurs terrines de production. On cueille à présent les apothécies lorsque l'on juge, par expérience, qu'elles ont atteint leur diamètre maximal, la taille du stipe donnant très tôt une idée de celle que peut atteindre l'apothécie.

Une amélioration considérable de la production d'ascospores consisterait à récolter les ascospores durant la vie entière des apothécies. On aboutirait ainsi à plusieurs millions d'ascospores par apothécie. Cela ne sera possible que lorsque l'on mettra au point une technique qui permettra à la fois une excellente sporulation des apothécies, la récolte continue des ascos- pores projetées et leur non-germination sur le support de récolte. Les appareillages que nous avons essayés dans ce but ne nous ont pas donné satisfaction. Les divers types d'aspirateurs de spores en continu, placés aussi bien sur les cristallisoirs contenant des apothécies séparées ou non de leurs sclérotes, qu'au-dessus des apothécies produites en «miniserres", entraînent en chambre de culture la dessication rapide des apothécies si l'on ne veille pas à conserver un air saturé d'eau. Mais si l'humidité est très forte, les ascospores récoltées germent rapidement et ne peuvent donc être conservées en vue de contaminations artificielles ultérieures.

D'autre part, la récolte des spores par une dépression ou une aspiration momentanées (Tourvieille et al, 1978 et Pierre, Cetiom, 1989, comm pers) si elle est pratiquée avec succès sur de petits lots d'apothécies de $S$ sclerotiorum en vue d'une utilisation immédiate des ascospores, s'est révélée inadaptée à nos conditions de production des apothécies en "Miniserres". Elle conduit en effet à perdre la majorité des ascospores, projetées sous forme de nuage, et celles que l'on récupère, humides, se conservent mal.

Les observations en chambre de culture et surtout les captures d'ascospores faites à l'extérieur apportent des précisions sur l'épidémiologie de la sclérotiniose des légumineuses fourragères. II est probable en effet qu'en culture de trèfle ou de luzerne, $S$ trifoliorum projette ses ascospores de la même façon que celle que nous avons montrée pour des apothécies placées hors de tout couvert végétal. Nous considérons que les conclusions auxquelles nous avons abouti reflètent assez bien la réalité, car elles sont en accord avec ce que nous connaissons, par d'autres expérimentations, de cette maladie (Raynal, 1985).

Cependant, il est légitime de penser qu'un couvert végétal favorise, notamment l'après-midi en conditions anticycloniques, une hygrométrie plus élevée au niveau des apothécies que celle de nos essais. II se peut donc que, lors de telles conditions météorologiques, une projection d'ascospores plus abondante que celle que nous avons mise en évidence puisse avoir lieu au champ. D'autre part, dans les conditions naturelles, les apothécies sont assez souvent dissimulées sous les parties basses des végétaux. Par temps humide ou pluvieux, on peut penser qu'elles restent plus longtemps mouillées que des apothécies soumises à une ventilation naturelle ou au courant d'air permanent dû à l'aspira- 
tion d'un capteur de spores. II se peut, de ce fait, que leur capacité à sporuler soit modifiée. De même, les apothécies non abritées, qui peuvent se ressuyer plus vite mais, qui, en contrepartie reçoivent directement les gouttes de pluie et le rayonnement solaire, ne fonctionnent peut-être pas de façon absolument identique à celles qui sont plus ou moins protégées par un capteur.

II s'ensuit que les captures que nous avons faites ne peuvent prétendre représenter la complexité de l'ensemble des situations du champ. Les résultats obtenus constituent cependant une base sûre pour d'autres études, qui pourraient s'intéresser par exemple à la dissémination éolienne des ascospores, non envisagée dans les travaux que nous rapportons, mais qui a fait l'objet d'études approfondies chez $S$ sclerotiorum (Suzui et Kobayashi, 1972; Abawi et Grogan, 1979; Williams et Stelfox, 1979).

Une vue d'ensemble sur la sclérotiniose de la luzerne et surtout du trèfle violet montre qu'elle dépend du cultivar, de l'itinéraire cultural (date de semis notamment), de la quantité d'inoculum disponible pour assurer les contaminations, et des conditions climatiques qui favoriseront ou non la constitution de l'inoculum et l'évolution de la maladie. Pour l'inoculum, sa quantité dépend d'abord de l'âge de la culture, qui définit le nombre de sclérotes présents dans le sol et, par là, l'abondance des apothécies dont la différenciation automnale est soumise au climat. La température et surtout le potentiel hydrique du sol seront alors déterminants (Raynal, 1987), ce dernier facteur ayant également été reconnu prépondérant pour $S$ sclerotiorum (Teo et Morrall, 1985). Une fois les apothécies constituées, leur maturation, leur durée de vie et la quantité d'ascospores projetées dépendront de la température, ainsi que de I'humidité relative de l'air et du potentiel hydrique du sol. On conçoit donc qu'en climat tempéré, la sclérotiniose des légumineuses fourragères soit particulièrement grave au cours des années à automne et hiver doux et humides et, a contrario, peu importante en cas de sécheresse automnale et d'hiver froid.

\section{REMERCIEMENTS}

Nous remercions vivement Madame C Lamarque et le Dr RAA Morrall pour leurs remarques et informations qui nous ont permis de préciser certains résultats.

\section{RÉFÉRENCES}

Abawi GS, Grogan RG (1979) Epidemiology of diseases caused by Sclerotinia species. Phytopathology 69, 899-904

Hirst JM (1952) An automatic volumetric spore trap. Ann Appl Biol 39, 257-265

Krüger W (1975) Die Beeinflussung der Apothezien und Ascosporen - Entwicklung des Rapskrebserregers Sclerotinia sclerotiorum (Lib) de Bary durch Umwelt faktoren. Z Pflanzenkrankh Pflanzensch 82, 101-108

Lamarque C, Rapilly F (1981) Conditions nécessaires à la contamination du tournesol par les ascospores de Sclerotinia sclerotiorum (Lib) de Bary. Application à la prévention des épidémies locales. Inf Tech CETIOM 75, 4-6

Newton HC, Sequeira L (1972) Ascospores as the primary infective propagule of Sclerotinia sclerotiorum in Wisconsin. Plant Dis Rep 56, 798-802

Rapilly F (1970) Réalisation d'un appareil automatique de capture des spores présentes dans l'air. Ann Phytopathol 2, 259-262

Raynal G (1983) Production au laboratoire d'apothécies de Sclerotinia trifoliorum Eriks pour l'évaluation de la résistance du trèfle violet à la sclérotiniose. Agronomie 3, 369-373

Raynal G (1985) Observations sur le comportement variétal au champ du trèfle violet vis-à-vis de la sclérotiniose et sur l'épidémiologie de la maladie. Fourrages 101, 85-104

Raynal G (1987) Facteurs agissant sur la formation des apothécies de Sclerotinia trifoliorum Eriks en conditions contrôlées. Agronomie 7, 715-725

Raynal G, Clinkspoor H (1988) Épidémiologie de la quenouille du dactyle et méthodes de lutte prophylactique envisageables. In: Ite Conf intern sur les maladies des plantes. Bordeaux, 8-10 nov, Annales ANPP, T2, 1049-1056

Schwartz HF, Steadman JR (1978) Factors affecting sclerotium population of and apothecium production by Sclerotinia sclerotiorum. Phytopathology 68, 383-388

Steadman JR (1983) White mold, a serious yieldlimiting disease of bean. Plant Disease 67, 346350

Suzui T, Kobayashi T (1972) Dispersal of ascospores of Sclerotinia sclerotiorum (Lib) De Bary on kidney bean plants. I. Dispersal of ascospores from a point source of apothecia. Res Bull Hokkaido Nat Agric Exp Station 101, 137-151

Taris B (1968) Contribution à l'étude des rouilles des Populus observées en France. Ann Epiphyties 19, 5-54

Teo BK, Morrall RAA (1985) Influence of matric potentials on carpogenic germination of sclerotia of Scle- 
rotinia sclerotirum. I. Development of an inclined box technique to observe apothecium production. Can J Plant Pathol 7, 359-364

Tourvieille de Labrouhe D, Guillaumin JJ, Vear F, Lamarque $C$ (1978) Rôle des ascospores dans l'infection du tournesol par Sclerotinia sclerotiorum (Lib) De By. Ann Phytopathol 10, 417-431
Valleau WD, Fergus EN, Henson L (1933) Resistance of red clovers to Sclerotinia trifoliorum Eriks and infection studies. Kentucky Agric Exp Sta Res Bull 341, 116-131

Williams JR, Stelfox D (1979) Dispersal of ascospores of Sclerotinia sclerotiorum in relation to Sclerotinia stem rot of rapeseed. Plant Dis Reptr 63, 395-399 